Pure and Applied Mathematics Quarterly

Volume 10, Number 2

(Special Issue: In memory of

Andrey Todorov, Part 3 of 3 )

$223-243,2014$

\title{
Goodness of Canonical Metrics on the Moduli Space of Riemann Surfaces
}

\author{
Kefeng Liu, Xiaofeng Sun and Shing-Tung Yau \\ Dedicated to Professor Andrey Todorov
}

\begin{abstract}
In this paper we will show the Mumford goodness of the metrics on the logarithmic tangent bundle of the moduli spaces of curves induced by the Ricci and the perturbed Ricci metrics. It follows from these estimates that the Ricci metric can be extended to the Deligne-Mumford compactification of the moduli spaces.
\end{abstract}

Keywords: canonical metrics, Mumford goodness, moduli spaces, natuality.

\section{Contents}

1. Introduction 224

2. Mumford Goodness and Canonical Metrics on the Moduli Spaces 225

3. Goodness of the Ricci and perturbed Ricci Metrics 232

4. Naturality of the Ricci Metric 239

$\begin{array}{ll}\text { References } & 242\end{array}$

Received December 6, 2013. 


\section{INTRODUCTION}

This paper is dedicated to our friend, professor Andrey Todorov. In this paper we study the Mumford goodness of various metrics on the logarithmic tangent bundle over the moduli space $\mathcal{M}_{g}$ of hyperbolic Riemann surfaces which are induced by canonical metrics on $\mathcal{M}_{g}$. By the Chern-Weil theory we know that for a Hermitian vector bundle $(E, h)$ over a compact complex manifold $X$, the $k$-th Chern form $c_{k}(E, h)$ represents the $k$-th Chern class of $E$. However, this is no longer true in general if $X$ is noncompact. In order to generalize the proportionality theorem of Hirzebruch, in [8] introduced the goodness condition on Hermitian metrics on holomorphic vector bundles over quasi-projective manifolds. These good metrics have desired asymptotics and the Chern-Weil theory still hold in the sense of currents. As we know that the moduli space $\mathcal{M}_{g}$ is not locally symmetric with respect to these canonical metrics. However $\mathcal{M}_{g}$ share many properties of locally symmetric spaces and the Mumford goodness of the canonical metrics is a strong evidence.

To setup notation we let $\mathcal{M}_{g}$ be the moduli space of Riemann surfaces of genus $g \geq 2$ and let $\overline{\mathcal{M}}_{g}$ be its Deligne-Mumford compactification where $D=\overline{\mathcal{M}}_{g} \backslash$ $\mathcal{M}_{g}$ be the compactification divisor. Let $\bar{E}=T_{\overline{\mathcal{M}}_{g}}^{1,0}(-\log D)$ be the logarithmic tangent bundle over $\overline{\mathcal{M}}_{g}$ and let $E=\left.\bar{E}\right|_{\mathcal{M}_{g}}$. The paper is organized as follows. In Section 2 we will review the definition and basis properties of good metrics in the sense of Mumford. We will also discuss the basic properties of the WeilPetersson, Ricci and perturbed Ricci metrics on the moduli space $\mathcal{M}_{g}$. In Section 3 we prove that the Ricci and perturbed Ricci metrics are good:

Theorem 1.1. Let $\tau$ be the Ricci metric, let $\tau_{c}$ be the perturbed Ricci metric and let $\widetilde{\tau}$ and $\widetilde{\tau}_{c}$ be the Hermitian metrics on $E$ induced by $\tau$ and $\tau_{c}$ respectively. Then $\widetilde{\tau}$ and $\widetilde{\tau}_{c}$ are good in the sense of Mumford.

The proof of this theorem is based on a refined version of the asymptotic analysis in [4] and [5].

Since each irreducible component of $D^{\prime} \subset D$ is a moduli space of Riemann surfaces with punctures, there is a natural Weil-Petersson metric on $D^{\prime}$. It is well known that the Weil-Petersson metric is natural in the sense that the WP metric on $\mathcal{M}_{g}$ can be extended to each $D^{\prime}$ and coincides with the WP metric on $D^{\prime}$. In Section 4 we prove the naturality of the Ricci metric: 
Theorem 1.2. The Ricci metric on $\mathcal{M}_{g}$ can be extended naturally to $D$ and coincide with the Ricci metric on each component of $D$.

\section{Mumford Goodness and Canonical Metrics on the Moduli Spaces}

In this section we first recall the Mumford goodness of singular Hermitian metrics. See [8] for details. We will use the following notations: let $\Delta_{r}$ be the open disk in $\mathbb{C}$ with radius $r$, let $\Delta=\Delta_{1}, \Delta_{r}^{*}=\Delta_{r} \backslash\{0\}$ and $\Delta^{*}=\Delta \backslash\{0\}$.

Let $\bar{X}$ be a projective manifold of complex dimension $\operatorname{dim}_{\mathbb{C}} \bar{X}=n$. Let $D \subset \bar{X}$ be a divisor with simple normal crossings and let $X=\bar{X} \backslash D$ be a Zariski open manifold. For each point $p \in D$ we can find a coordinate chart $\left(U, z_{1}, \cdots, z_{n}\right)$ around $p$ in $\bar{X}$ such that $U \cong \Delta^{n}$ and $V=U \cap X \cong\left(\Delta^{*}\right)^{m} \times \Delta^{n-m}$. We assume that $U \cap D$ is defined by the equation $z_{1} \cdots z_{m}=0$. We let $U(r) \cong \Delta_{r}^{n}$ for $0<r<1$ and let $V(r)=U(r) \cap X$.

On the chart $V$ of $X$ we can define a local Poincaré metric:

$$
\omega_{l o c}=\frac{\sqrt{-1}}{2} \sum_{i=1}^{m} \frac{d z_{i} \wedge d \bar{z}_{i}}{2\left|z_{i}\right|^{2}\left(\log \left|z_{i}\right|\right)^{2}}+\frac{\sqrt{-1}}{2} \sum_{i=m+1}^{n} d z_{i} \wedge d \bar{z}_{i} .
$$

Now we cover $D \subset \bar{X}$ by such coordinate charts $U_{1}, \cdots, U_{q}$ and let $V_{i}=U_{i} \cap X$. We choose coordinate $z_{1}^{i}, \cdots, z_{n}^{i}$ such that $D \cap U_{i}$ is given by $z_{1}^{i} \cdots z_{m_{i}}^{i}=0$. A Kähler metric $\omega_{g}$ on $X$ has Poincaré growth if for each $1 \leq i \leq q$ there are constants $0 \leq r_{i}<1$ and $0<c_{i}<C_{i}$ such that $\left.\omega_{g}\right|_{V_{i}\left(r_{i}\right)}$ is equivalent to the local Poincaré metric $\omega_{l o c}^{i}$ :

$$
c_{i} \omega_{l o c}^{i} \leq\left.\omega_{g}\right|_{V_{i}\left(r_{i}\right)} \leq C_{i} \omega_{l o c}^{i} .
$$

We note that there exists a Kähler metric on $X$ with Poincaré growth since $\bar{X}$ is projective. In [8] Mumford defined good differential forms on $X$ :

Definition 2.1. Let $\eta \in A^{p}(X)$ be a smooth p-form. Then $\eta$ has Poincaré growth if for each $1 \leq i \leq q$ there exists a constant $a_{i}>0$ such that for each point $s \in V_{i}\left(\frac{1}{2}\right)$ and tangent vectors $t_{1}, \cdots, t_{p} \in T_{s} X$ one has

$$
\left|\eta\left(t_{1}, \cdots, t_{p}\right)\right|^{2} \leq a_{i} \prod_{j=1}^{p} \omega_{l o c}^{i}\left(t_{j}, t_{j}\right) .
$$

The p-form $\eta$ is good if and only if both $\eta$ and $d \eta$ have Poincaré growth. 
Remark 2.1. It is easy to see that the above definition does not depend on the choice of the cover $\left(U_{1}, \cdots, U_{q}\right)$ but it does depend on the compactification $\bar{X}$ of $X$. Furthermore, a direct computation shows that a p-form $\eta \in A^{p}(X)$ has Poincaré growth if and only if $\|\eta\|_{g}<\infty$ where $\omega_{g}$ is any Kähler metric on $X$ with Poincaré growth. It follows that if $\eta_{1} \in A^{p}(X)$ and $\eta_{2} \in A^{q}(X)$ have Poincaré growth, then $\eta_{1} \wedge \eta_{2}$ also has Poincaré growth.

Now we consider a holomorphic vector bundle $\bar{E}$ or rank $r$ over $\bar{X}$. Let $E=$ $\left.\bar{E}\right|_{X}$ and let $h$ be a Hermitian metric on $E$. According to [8] we have

Definition 2.2. The Hermitian metric $h$ is good if for any point $x \in D$, assume $x \in U_{i}$ for some $i$, and any basis $e_{1}, \cdots, e_{r}$ of $\left.\bar{E}\right|_{U_{i}\left(\frac{1}{2}\right)}$, if we let $h_{\alpha \bar{\beta}}=h\left(e_{\alpha}, e_{\beta}\right)$ then there exist positive constants $c_{i}, d_{i}$ such that

(1) $\left|h_{\alpha \bar{\beta}}\right|,(\operatorname{det} h)^{-1} \leq c_{i}\left(\sum_{j=1}^{m_{i}} \log \left|z_{j}\right|\right)^{2 d_{i}} ;$

(2) the 1 -forms $\left(\partial h \cdot h^{-1}\right)_{\alpha \beta}$ are good on $V_{i}\left(\frac{1}{2}\right)$.

Remark 2.2. We note that the definition is independent of the choice of local basis $e_{1}, \cdots, e_{r}$.

The following important properties of forms with Poincaré growth and good metrics are proved in [8].

Proposition 2.1. Let $X, \bar{X}, E$ and $\bar{E}$ be as above. Then

(1) If $\eta \in A^{p}(X)$ be a form with Poincaré growththen $\eta$ defines a p-current on $\bar{X}$. Furthermore, if $\eta$ is good then $d[\eta]=[d \eta]$.

(2) Let $h$ be a Hermitian metric on $E$. Then there is at most one extension of $E$ to $\bar{X}$ for which $h$ is good. Furthermore, if $h$ is a good metric on $E$, then the Chern forms $c_{k}(E, h)$ are good and the current $\left[c_{k}(E, h)\right]=$ $c_{k}(\bar{E}) \in H^{2 k}(\bar{X})$.

This theorem allows us to compute the Chern classes by using Chern forms of a singular good metric.

Now we look at the case of logarithmic tangent bundle. In the following we let

$$
\bar{E}=T_{\bar{X}}(-\log D)
$$

and let $E=\left.\bar{E}\right|_{X}$. Let $U$ be one of the charts $U_{i}$ described above and assume $D \cap U$ is given by $z_{1} \cdots z_{m}=0$. Let $V=V_{i}=U_{i} \cap X$. In this case a local frame 
of $\bar{E}$ restricting to $V$ is given by

$$
e_{1}=z_{1} \frac{\partial}{\partial z_{1}}, \cdots, e_{m}=z_{m} \frac{\partial}{\partial z_{m}}, e_{m+1}=\frac{\partial}{\partial z_{m+1}}, \cdots, e_{n}=\frac{\partial}{\partial z_{n}}
$$

Let $g$ be any Kähler metric on $X$. It induces a Hermitian metric $\widetilde{g}$ on $E$. In local coordinate $z=\left(z_{1}, \cdots, z_{n}\right)$ we have

$$
\widetilde{g}_{i \bar{j}}= \begin{cases}z_{i} \bar{z}_{j} g_{i \bar{j}} & \text { if } \quad i, j \leq m \\ z_{i} g_{i \bar{j}} & \text { if } \quad i \leq m<j \\ \bar{z}_{j} g_{i \bar{j}} & \text { if } \quad j \leq m<i \\ g_{i \bar{j}} & \text { if } \quad i, j>m .\end{cases}
$$

In the following we denote by $\partial_{i}$ the partial derivative $\frac{\partial}{\partial z_{i}}$. Let

$$
\Gamma_{i k}^{p}=g^{p \bar{q}} \partial_{i} g_{k \bar{q}}
$$

be the Christoffell symbol of the Kähler metric $g$ and let

$$
R_{i k \bar{l}}^{p}=g^{p \bar{j}} R_{i \bar{j} k \bar{l}}=g^{p \bar{j}}\left(-\partial_{k} \partial_{\bar{l}} g_{i \bar{j}}+g^{s \bar{t}} \partial_{k} g_{i \bar{t}} \partial_{\bar{l}} g_{s \bar{j}}\right)
$$

be the curvature of $g$. We let

$$
\rho_{i}=\left\{\begin{array}{lll}
z_{i} & \text { if } & i \leq m \\
1 & \text { if } & i>m
\end{array}\right.
$$

and

$$
\Lambda_{i}=\left\{\begin{array}{lll}
\frac{-1}{\left|z_{i}\right| \log \left|z_{i}\right|} & \text { if } \quad i \leq m \\
1 & \text { if } \quad i>m
\end{array}\right.
$$

Now we give an equivalent local condition of the metric $\widetilde{g}$ on $E$ induced by the Kähler metric $g$ to be good. We have 
Proposition 2.2. The metric $\widetilde{g}$ on $E$ induced by $g$ is good on $V\left(\frac{1}{2}\right)$ if and only if

$$
\begin{aligned}
& \left|\widetilde{g}_{i \bar{j}}\right|,\left|z_{1} \cdots z_{m}\right|^{-2} \operatorname{deg}(g)^{-1} \leq c\left(\sum_{i=1}^{m} \log \left|z_{i}\right|\right)^{2 d} \text { for some constants } c, d>0 \\
& \left|\frac{\rho_{i}}{\rho_{k}} \Gamma_{i p}^{k}\right|=O\left(\Lambda_{p}\right) \quad \text { for all } 1 \leq i, k, p \leq n \text { except } i=k=p \\
& \left|\frac{1}{z_{i}}+\Gamma_{i i}^{i}\right|=O\left(\Lambda_{i}\right) \quad \text { if } i \leq m \\
& \left|\frac{\rho_{i}}{\rho_{k}} R_{i p \bar{q}}^{k}\right|=O\left(\Lambda_{p} \Lambda_{q}\right) .
\end{aligned}
$$

Proof. We note that the first inequality of (2.5) follows from the first condition of Definition 2.2 and formula (2.2) directly.

Now we let $A=\left[\partial \widetilde{g} \cdot \widetilde{g}^{-1}\right]$ be the connection matrix. By direct computations we know

(2.6) $\quad A_{i}^{k}=\left\{\begin{array}{ll}\sum_{p} \frac{\rho_{i}}{\rho_{k}} \Gamma_{i p}^{k} d z_{p} & \text { if } \quad i \neq k \text { or } i=k \geq m+1 \\ \sum_{p \neq i} \Gamma_{i p}^{i} d z_{p}+\left(\frac{1}{z_{i}}+\Gamma_{i i}^{i}\right) d z_{i} & \text { if } \quad i=k \leq m\end{array}\right.$.

The second and third inequalities of (2.5) are equivalent to the fact that the local one forms $A_{i}^{k}$ have finite norm with respect to the local Poincaré metric as defined by formula (2.1). By Remark 2.1 we know that the second and third inequalities of (2.5) are equivalent to the fact that $A_{i}^{k}$ has Poincaré growth.

Now we looking at the last inequality of (2.5). We have $d A=\bar{\partial} A-A \wedge A$. By Remark 2.1 we know that if each entry of $A$ has Poincaré growth, then each entry of $A \wedge A$ has Poincaré growth. Thus each entry of $d A$ has Poincaré growth if and only if each entry of $\bar{\partial} A$ has Poincaré growth. By formula (2.6) we know that $(\bar{\partial} A)_{i}^{k}=\frac{\rho_{i}}{\rho_{k}} R_{i p \bar{q}}^{k} d z_{p} \wedge d \bar{z}_{q}$. Thus the last inequality of (2.5) is equivalent to the fact that each entry of $d A$ has Poincaré growth. The proposition follows from Definition 2.2.

Next we recall several canonical metrics on the moduli space $\mathcal{M}_{g}$ especially those introduced in [4] and their properties. We assume $g \geq 2$ and let $n=$ $3 g-3=\operatorname{dim}_{\mathbb{C}} \mathcal{M}_{g}$. For each point $s \in \mathcal{M}_{g}$ we let $X_{s}$ be the corresponding 
Riemann surface. By the uniformization theorem we know that there exist a unique Kähler-Einstein metric $\lambda$ on $X_{s}$ :

$$
\partial_{z} \partial_{\bar{z}} \log \lambda=\lambda
$$

We assume $s \in \mathcal{M}_{g}$ is a smooth point and let $B \subset \mathcal{M}_{g}$ be an open neighborhood. We consider the harmonic Kodaira-Spencer map

$$
K S: T_{s}^{1,0} B \rightarrow \mathbb{H}^{0,1}\left(X_{s}, T_{X_{s}}^{1,0}\right)
$$

where $\mathbb{H}^{0,1}\left(X_{s}, T_{X_{s}}^{1,0}\right)$ is the space of harmonic Beltrami differentials with respect to the Kähler-Einstein metric on $X_{s}$. Let $t=\left(t_{1}, \cdots, t_{n}\right)$ be any holomorphic coordinates on $B$. We let $B_{i}=K S\left(\frac{\partial}{\partial t_{i}}\right)$. Then Weil-Petersson metric at 0 is defined as

$$
h_{i \bar{j}}(s)=\int_{X_{s}} B_{i} \bar{B}_{j} d v
$$

where $d v$ is the Kähler-Einstein volume form.

The curvature of the WP metric is computed by Wolpert [10]. Let $f_{i \bar{j}}=$ $B_{i} \bar{B}_{j} \in C^{\infty}\left(X_{s}\right)$, let $\square=-\lambda^{-1} \partial_{z} \partial_{\bar{z}}$ be the Hodge Laplacian and let $e_{i \bar{j}}=$ $(\square+1)^{-1}\left(f_{i \bar{j}}\right)$. The curvature of the WP metric is

$$
R_{i \bar{j} k \bar{l}}=-\int_{X_{s}}\left(e_{i \bar{j}} f_{k \bar{l}}+e_{i \bar{l}} f_{k \bar{j}}\right) d v .
$$

It follows from this formula that the curvature of the WP metric has various negativity properties. In particular the Ricci curvature of the WP metric is negative definite. However, the curvature of the WP metric has no lower bound and this metric is incomplete.

In [4] we introduced the Ricci metric

$$
\omega_{\tau}=-\operatorname{Ric}\left(\omega_{W P}\right)
$$

and the perturbed Ricci metric

$$
\omega_{C}=\omega_{\tau}+C \omega_{W P}
$$

for any constant $C>0$. It turns out that the Ricci and perturbed Ricci metrics have many nice properties.

Similar to formula (2.7) we can establish integral formulae for the curvature of the Ricci and perturbed Ricci metrics. These curvature formulae are crucial 
in estimating the asymptotic of these metrics and their curvature. To establish these formulae, we need to introduce some operators. We let

$$
P: C^{\infty}\left(X_{s}\right) \rightarrow A^{1,0}\left(T_{X_{s}}^{0,1}\right)
$$

be the operator defined by

$$
\left.f \mapsto \partial\left(\omega_{K E}^{-1}\right\lrcorner \partial f\right) .
$$

In local coordinate we have $P(f)=\partial_{z}\left(\lambda^{-1} \partial_{z} f\right) d z \otimes \partial_{\bar{z}}$. For each $1 \leq k \leq n$ we let

$$
\xi_{k}: C^{\infty}\left(X_{s}\right) \rightarrow C^{\infty}\left(X_{s}\right)
$$

be the operator defined by

$$
\left.f \mapsto \bar{\partial}^{*}\left(B_{k}\right\lrcorner \partial f\right)=-B_{k} \cdot P(f) .
$$

In local coordinate we have $\xi_{k}(f)=-\lambda^{-1} \partial_{z}\left(A_{k} \partial_{z} f\right)$ where $B_{k}=A_{k} d \bar{z} \otimes \frac{\partial}{\partial z}$. Finally for any $1 \leq k, l \leq n$ we define the operator

$$
Q_{k \bar{l}}: C^{\infty}\left(X_{s}\right) \rightarrow C^{\infty}\left(X_{s}\right)
$$

by

$$
Q_{k \bar{l}}(f)=\bar{P}\left(e_{k \bar{l}}\right) P(f)-2 f_{k \bar{l}} \square f+\lambda^{-1} \partial_{z} f_{k \bar{l}} \partial_{\bar{z}} f
$$

These operators are commutators of various classical operators on $X_{s}$. See [4] for details. Now we recall the curvature formulae of the Ricci and perturbed Ricci metrics established in [4]. For convenience, we introduce the symmetrization operator.

Definition 2.3. Let $U$ be any quantity which depends on indices $i, k, \alpha, \bar{j}, \bar{l}, \bar{\beta}$. The symmetrization operator $\sigma_{1}$ is defined by taking the summation of all orders of the triple $(i, k, \alpha)$. That is

$$
\begin{aligned}
\sigma_{1}(U(i, k, \alpha, \bar{j}, \bar{l}, \bar{\beta}))= & U(i, k, \alpha, \bar{j}, \bar{l}, \bar{\beta})+U(i, \alpha, k, \bar{j}, \bar{l}, \bar{\beta})+U(k, i, \alpha, \bar{j}, \bar{l}, \bar{\beta}) \\
& +U(k, \alpha, i, \bar{j}, \bar{l}, \bar{\beta})+U(\alpha, i, k, \bar{j}, \bar{l}, \bar{\beta})+U(\alpha, k, i, \bar{j}, \bar{l}, \bar{\beta}) .
\end{aligned}
$$

Similarly, $\sigma_{2}$ is the symmetrization operator of $\bar{j}$ and $\bar{\beta}$ and $\widetilde{\sigma_{1}}$ is the symmetrization operator of $\bar{j}, \bar{l}$ and $\bar{\beta}$.

Now we can state the curvature formulae. 
Theorem 2.1. Let $s_{1}, \cdots, s_{n}$ be local holomorphic coordinates at $s \in \mathcal{M}_{g}$ and let $\widetilde{R}_{i \bar{j} k \bar{l}}$ be the curvature of the Ricci metric. Then at $s$, we have

$$
\begin{aligned}
& \widetilde{R}_{i \bar{j} k \bar{l}}= \\
& -h^{\alpha \bar{\beta}}\left\{\sigma_{1} \sigma_{2} \int_{X_{s}}\left\{(\square+1)^{-1}\left(\xi_{k}\left(e_{i \bar{j}}\right)\right) \bar{\xi}_{l}\left(e_{\alpha \bar{\beta}}\right)+(\square+1)^{-1}\left(\xi_{k}\left(e_{i \bar{j}}\right)\right) \bar{\xi}_{\beta}\left(e_{\alpha \bar{l}}\right)\right\} d v\right\} \\
& -h^{\alpha \bar{\beta}}\left\{\sigma_{1} \int_{X_{s}} Q_{k \bar{l}}\left(e_{i \bar{j}}\right) e_{\alpha \bar{\beta}} d v\right\} \\
& \left.+\tau^{p \bar{q}} h^{\alpha \bar{\beta}} h^{\gamma \bar{\delta}}\left\{\sigma_{1} \int_{X_{s}} \xi_{k}\left(e_{i \bar{q}}\right) e_{\alpha \bar{\beta}} d v\right\}\left\{\widetilde{\sigma}_{1} \int_{X_{s}} \bar{\xi}_{l}\left(e_{p \bar{j}}\right) e_{\gamma \bar{\delta}}\right) d v\right\} \\
& +\tau_{p \bar{j}} h^{p \bar{q}} R_{i \bar{q} k \bar{l}} .
\end{aligned}
$$

Theorem 2.2. Let $\widetilde{\tau}_{i \bar{j}}=\tau_{i \bar{j}}+C h_{i \bar{j}}$ where $\tau$ and $h$ are the Ricci and WP metrics respectively where $C>0$ is a constant. Let $P_{i \bar{j} k \bar{l}}$ be the curvature of the perturbed Ricci metric. Then we have

$$
\begin{aligned}
& P_{i \bar{j} k \bar{l}}= \\
& -h^{\alpha \bar{\beta}}\left\{\sigma_{1} \sigma_{2} \int_{X_{s}}\left\{(\square+1)^{-1}\left(\xi_{k}\left(e_{i \bar{j}}\right)\right) \bar{\xi}_{l}\left(e_{\alpha \bar{\beta}}\right)+(\square+1)^{-1}\left(\xi_{k}\left(e_{i \bar{j}}\right)\right) \bar{\xi}_{\beta}\left(e_{\alpha \bar{l}}\right)\right\} d v\right\} \\
& -h^{\alpha \bar{\beta}}\left\{\sigma_{1} \int_{X_{s}} Q_{k \bar{l}}\left(e_{i \bar{j}}\right) e_{\alpha \bar{\beta}} d v\right\} \\
& \left.+\widetilde{\tau}^{p \bar{q}} h^{\alpha \bar{\beta}} h^{\gamma \bar{\delta}}\left\{\sigma_{1} \int_{X_{s}} \xi_{k}\left(e_{i \bar{q}}\right) e_{\alpha \bar{\beta}} d v\right\}\left\{\widetilde{\sigma}_{1} \int_{X_{s}} \bar{\xi}_{l}\left(e_{p \bar{j}}\right) e_{\gamma \bar{\delta}}\right) d v\right\} \\
& +\tau_{p \bar{j}} h^{p \bar{q}} R_{i \bar{q} k \bar{l}}+C R_{i \bar{j} k \bar{l}} .
\end{aligned}
$$

In order to estimate the connection forms of the metrics on $E$ induced by the Ricci and perturbed Ricci metrics we also need to following formula about the first order derivatives of the Ricci metric.

Theorem 2.3. Let $\Gamma_{i k}^{p}$ be the Christoffell symbol of the Weil-Petersson metric. Then

$$
\partial_{k} \tau_{i \bar{j}}=h^{\alpha \bar{\beta}}\left\{\sigma_{1} \int_{X_{s}}\left(\xi_{k}\left(e_{i \bar{j}}\right) e_{\alpha \bar{\beta}}\right) d v\right\}+\tau_{p \bar{j}} \Gamma_{i k}^{p}
$$

Now we look at properties of the Ricci and perturbed Ricci metrics. Here we collect the important ones from [4] and [5]. 
Theorem 2.4. For any $g \geq 2$ let $\omega_{\tau}$ and $\omega_{C}$ be the Ricci and perturbed Ricci metrics on $\mathcal{M}_{g}$. Then

(1) The Ricci and perturbed Ricci metrics are complete and have Poincaré growth. In particular the moduli space equipped with any of these metrics has finite volume.

(2) These metrics are equivalent to the Kähler-Einstein metric, the Kobayashi metric, the Carathédory metric, the Bergman metric and the McMullen metric on $\mathcal{M}_{g}$.

(3) The curvatures of the Ricci and perturbed Ricci metrics are bounded. Furthermore, on the Teichmüller space $\mathcal{T}_{g}$ the injectivity radius of these metrics is bounded.

(4) With suitable choice of $C$ the holomorphic sectional curvature and the Ricci curvature of the perturbed Ricci metric $\omega_{C}$ are bounded from above and below by negative constants.

\section{Goodness of the Ricci and perturbed Ricci Metrics}

In this section we show that the metrics on $E$ induced by the Ricci and perturbed Ricci metrics are good in the sense of Mumford.

Theorem 3.1. Let $\tau$ and $\tau_{C}$ be the Ricci and perturbed Ricci metrics on $\mathcal{M}_{g}$ and let $\widetilde{\tau}$ and $\widetilde{\tau}_{C}$ be the metrics on $E=\left.T_{\overline{\mathcal{M}}_{g}}^{1,0}(-\log D)\right|_{\mathcal{M}_{g}}$ induced by $\tau$ and $\tau_{C}$. Then $\widetilde{\tau}$ and $\widetilde{\tau}_{C}$ are good.

To prove this theorem we first describe the local pinching coordinates near the boundary of the moduli space due to the plumbing construction of Wolpert as well as the asymptotic of these metrics.

Let $\mathcal{M}_{g}$ be the moduli space of Riemann surfaces of genus $g \geq 2$ and let $\overline{\mathcal{M}}_{g}$ be its Deligne-Mumford compactification [2]. Each point $y \in \overline{\mathcal{M}}_{g} \backslash \mathcal{M}_{g}$ corresponds to a stable nodal surface $X_{y}$. A point $p \in X_{y}$ is a node if there is a neighborhood of $p$ which is isometric to the germ $\{(u, v)|u v=0| u,|| v \mid,<1\} \subset \mathbb{C}^{2}$.

We first recall the rs-coordinate on a Riemann surface defined by Wolpert in [12]. There are two cases: the puncture case and the short geodesic case. For the puncture case, we have a nodal surface $X$ and a node $p \in X$. Let $a, b$ be two punctures which are glued together to form $p$. 
Definition 3.1. A local coordinate chart $(U, u)$ near a is called rs-coordinate if $u(a)=0$ where $u$ maps $U$ to the punctured disc $0<|u|<c$ with $c>0$, and the restriction to $U$ of the Kähler-Einstein metric on $X$ can be written as $\frac{1}{2|u|^{2}(\log |u|)^{2}}|d u|^{2}$. The rs-coordinate $(V, v)$ near $b$ is defined in a similar way.

For the short geodesic case, we have a closed surface $X$, a closed geodesic $\gamma \subset X$ with length $l<c_{*}$ where $c_{*}$ is the collar constant.

Definition 3.2. A local coordinate chart $(U, z)$ is called rs-coordinate at $\gamma$ if $\gamma \subset U$ where $z$ maps $U$ to the annulus $c^{-1}|t|^{\frac{1}{2}}<|z|<c|t|^{\frac{1}{2}}$, and the KählerEinstein metric on $X$ can be written as $\frac{1}{2}\left(\frac{\pi}{\log |t|} \frac{1}{|z|} \csc \frac{\pi \log |z|}{\log |t|}\right)^{2}|d z|^{2}$.

By Keen's collar theorem [3], we have the following lemma:

Lemma 3.1. Let $X$ be a closed surface and let $\gamma$ be a closed geodesic on $X$ such that the length $l$ of $\gamma$ satisfies $l<c_{*}$. Then there is a collar $\Omega$ on $X$ with holomorphic coordinate $z$ defined on $\Omega$ such that

(1) $z$ maps $\Omega$ to the annulus $\frac{1}{c} e^{-\frac{2 \pi^{2}}{l}}<|z|<c$ for $c>0$;

(2) the Kähler-Einstein metric on $X$ restricted to $\Omega$ is given by

$$
\left(\frac{1}{2} u^{2} r^{-2} \csc ^{2} \tau\right)|d z|^{2}
$$

where $u=\frac{l}{2 \pi}, r=|z|$ and $\tau=u \log r$;

(3) the geodesic $\gamma$ is given by the equation $|z|=e^{-\frac{\pi^{2}}{l}}$.

We call such a collar $\Omega$ a genuine collar.

We notice that the constant $c$ in the above lemma has a lower bound such that the area of $\Omega$ is bounded from below. Also, the coordinate $z$ in the above lemma is rs-coordinate. In the following, we will keep the notations $u, r$ and $\tau$.

Now we describe the local manifold cover of $\overline{\mathcal{M}}_{g}$ near the boundary. We take the construction of Wolpert [12]. Let $X_{0,0}$ be a stable nodal surface corresponding to a codimension $m$ boundary point and let $p_{1}, \cdots, p_{m}$ be the nodes of $X_{0.0}$. The smooth part $X_{0}=X_{0,0} \backslash\left\{p_{1}, \cdots, p_{m}\right\}$ is a union of punctured Riemann surfaces. Fix the rs-coordinate charts $\left(U_{i}, \eta_{i}\right)$ and $\left(V_{i}, \zeta_{i}\right)$ at $p_{i}$ for $i=1, \cdots, m$ such that all the $U_{i}$ and $V_{i}$ are mutually disjoint.

Now pick an open set $U_{0} \subset X_{0}$ such that the intersection of each connected component of $X_{0}$ and $U_{0}$ is a nonempty relatively compact set and the intersection 
$U_{0} \cap\left(U_{i} \cup V_{i}\right)$ is empty for all $i$. We pick Beltrami differentials $\nu_{m+1}, \cdots, \nu_{n}$ which are supported in $U_{0}$ and span the tangent space at $X_{0}$ of the deformation space of $X_{0}$. For $s=\left(s_{m+1}, \cdots, s_{n}\right)$, let $\nu(s)=\sum_{i=m+1}^{n} s_{i} \nu_{i}$. We assume $|s|=\left(\sum\left|s_{i}\right|^{2}\right)^{\frac{1}{2}}$ small enough such that $|\nu(s)|<1$. The nodal surface $X_{0, s}$ is obtained by solving the Beltrami equation $\bar{\partial} w=\nu(s) \partial w$. Since $\nu(s)$ is supported in $U_{0},\left(U_{i}, \eta_{i}\right)$ and $\left(V_{i}, \zeta_{i}\right)$ are still holomorphic coordinates on $X_{0, s}$. Note that they are no longer rs-coordinates. By the theory of Alhfors and Bers [1] and Wolpert [12] we can assume that there are constants $\delta, c>0$ such that when $|s|<\delta, \eta_{i}$ and $\zeta_{i}$ are holomorphic coordinates on $X_{0, s}$ with $0<\left|\eta_{i}\right|<c$ and $0<\left|\zeta_{i}\right|<c$.

Now we assume $t=\left(t_{1}, \cdots, t_{m}\right)$ has small norm. We do the plumbing construction on $X_{0, s}$ to obtain $X_{t, s}$. We remove from $X_{0, s}$ the discs $0<\left|\eta_{i}\right| \leq \frac{\left|t_{i}\right|}{c}$ and $0<\left|\zeta_{i}\right| \leq \frac{\left|t_{i}\right|}{c}$ for each $i=1, \cdots, m$, and identify $\frac{\left|t_{i}\right|}{c}<\left|\eta_{i}\right|<c$ with $\frac{\left|t_{i}\right|}{c}<\left|\zeta_{i}\right|<c$ by the rule $\eta_{i} \zeta_{i}=t_{i}$. This defines the surface $X_{t, s}$. The tuple $\left(t_{1}, \cdots, t_{m}, s_{m+1}, \cdots, s_{n}\right)$ are the local pinching coordinates for the manifold cover of $\overline{\mathcal{M}}_{g}$. We call the coordinates $\eta_{i}$ (or $\zeta_{i}$ ) the plumbing coordinates on $X_{t, s}$ and the collar defined by $\frac{\left|t_{i}\right|}{c}<\left|\eta_{i}\right|<c$ the plumbing collar.

Remark 3.1. ¿From the estimate of Wolpert [11], [12] on the length of short geodesic, we have $u_{i}=\frac{l_{i}}{2 \pi} \sim-\frac{\pi}{\log \left|t_{i}\right|}$.

We also need the following version of the Schauder estimate proved by Wolpert in $[12]$.

Theorem 3.2. Let $X$ be a closed Riemann surface equipped with the unique Kähler-Einstein metric. Let $f$ and $g$ be smooth functions on $X$ such that $(\square+$ $1) g=f$. Then for any integer $k \geq 0$, there is a constant $c_{k}$ such that $\|g\|_{k+1} \leq$ $c_{k}\|f\|_{k}$.

Let $(t, s)=\left(t_{1}, \cdots, t_{m}, s_{m+1}, \cdots, s_{n}\right)$ be the pinching coordinates near $X_{0,0}$. For $|(t, s)|<\delta$, let $\Omega_{c}^{j}$ be the $j$-th genuine collar on $X_{t, s}$ which contains a short geodesic $\gamma_{j}$ with length $l_{j}$. Let $u_{j}=\frac{l_{j}}{2 \pi}, u_{0}=\sum_{j=1}^{m} u_{j}+\sum_{j=m+1}^{n}\left|s_{j}\right|, r_{j}=\left|z_{j}\right|$ and $\tau_{j}=u_{j} \log r_{j}$ where $z_{j}$ is the properly normalized rs-coordinate on $\Omega_{c}^{j}$ such that

$$
\Omega_{c}^{j}=\left\{z_{j}\left|c^{-1} e^{-\frac{2 \pi^{2}}{l_{j}}}<\right| z_{j} \mid<c\right\}
$$


¿From the above argument, we know that the Kähler-Einstein metric $\lambda$ on $X_{t, s}$, restrict to the collar $\Omega_{c}^{j}$, is given by

$$
\lambda=\frac{1}{2} u_{j}^{2} r_{j}^{-2} \csc ^{2} \tau_{j}
$$

For convenience, we let $\Omega_{c}=\cup_{j=1}^{m} \Omega_{c}^{j}$ and $R_{c}=X_{t, s} \backslash \Omega_{c}$. In the following, we may change the constant $c$ finitely many times, clearly this will not affect the estimates.

To estimate the WP, Ricci and perturbed Ricci metrics and their curvatures, the first step is to find all the harmonic Beltrami differentials $B_{1}, \cdots, B_{n}$ which correspond to the tangent vectors $\frac{\partial}{\partial t_{1}}, \cdots, \frac{\partial}{\partial s_{n}}$. In [7], Masur constructed $3 g-3$ regular holomorphic quadratic differentials $\psi_{1}, \cdots, \psi_{n}$ on the plumbing collars by using the plumbing coordinate $\eta_{j}$. These quadratic differentials correspond to the cotangent vectors $d t_{1}, \cdots, d s_{n}$.

However, it is more convenient to estimate the curvature if we use the rscoordinate on $X_{t, s}$ since we have the accurate form of the Kähler-Einstein metric $\lambda$ in this coordinate. In [9], Trapani used the graft metric constructed by Wolpert [12] to estimate the difference between the plumbing coordinate and rs-coordinate and described the holomorphic quadratic differentials constructed by Masur in the rs-coordinate. We collect Trapani's results (Lemma 6.2-6.5, [9]) in the following theorem:

Theorem 3.3. Let $(t, s)$ be the pinching coordinates on $\overline{\mathcal{M}}_{g}$ near $X_{0,0}$ which corresponds to a codimension $m$ boundary point of $\overline{\mathcal{M}}_{g}$. Then there exist constants $M, \delta>0$ and $1>c>0$ such that if $|(t, s)|<\delta$, then the $j$-th plumbing collar on $X_{t, s}$ contains the genuine collar $\Omega_{c}^{j}$. Furthermore, one can choose rs-coordinate $z_{j}$ on the collar $\Omega_{c}^{j}$ properly such that the holomorphic quadratic differential$s \psi_{1}, \cdots, \psi_{n}$ corresponding to the cotangent vectors $d t_{1}, \cdots, d s_{n}$ have the form $\psi_{i}=\varphi_{i}\left(z_{j}\right) d z_{j}^{2}$ on the genuine collar $\Omega_{c}^{j}$ for $1 \leq j \leq m$, where

(1) $\varphi_{i}\left(z_{j}\right)=\frac{1}{z_{j}^{2}}\left(q_{i}^{j}\left(z_{j}\right)+\beta_{i}^{j}\right)$ if $i \geq m+1$;

(2) $\varphi_{i}\left(z_{j}\right)=\left(-\frac{t_{j}}{\pi}\right) \frac{1}{z_{j}^{2}}\left(q_{j}\left(z_{j}\right)+\beta_{j}\right)$ if $i=j$;

(3) $\varphi_{i}\left(z_{j}\right)=\left(-\frac{t_{i}}{\pi}\right) \frac{1}{z_{j}^{2}}\left(q_{i}^{j}\left(z_{j}\right)+\beta_{i}^{j}\right)$ if $1 \leq i \leq m$ and $i \neq j$. 
Here $\beta_{i}^{j}$ and $\beta_{j}$ are functions of $(t, s), q_{i}^{j}$ and $q_{j}$ are functions of $\left(t, s, z_{j}\right)$ given by

$$
q_{i}^{j}\left(z_{j}\right)=\sum_{k<0} \alpha_{i k}^{j}(t, s) t_{j}^{-k} z_{j}^{k}+\sum_{k>0} \alpha_{i k}^{j}(t, s) z_{j}^{k}
$$

and

such that

$$
q_{j}\left(z_{j}\right)=\sum_{k<0} \alpha_{j k}(t, s) t_{j}^{-k} z_{j}^{k}+\sum_{k>0} \alpha_{j k}(t, s) z_{j}^{k}
$$

(1) $\sum_{k<0}\left|\alpha_{i k}^{j}\right| c^{-k} \leq M$ and $\sum_{k>0}\left|\alpha_{i k}^{j}\right| c^{k} \leq M$ if $i \neq j$;

(2) $\sum_{k<0}\left|\alpha_{j k}\right| c^{-k} \leq M$ and $\sum_{k>0}\left|\alpha_{j k}\right| c^{k} \leq M$;

(3) $\left|\beta_{i}^{j}\right|=O\left(\left|t_{j}\right|^{\frac{1}{2}-\epsilon}\right)$ with $\epsilon<\frac{1}{2}$ if $i \neq j$;

(4) $\left|\beta_{j}\right|=\left(1+O\left(u_{0}\right)\right)$.

Now we recall the asymptotic of the WP and Ricci metrics.

Theorem 3.4. Let $(t, s)$ be the pinching coordinates. Then the WP metric has asymptotic

(1) $h^{i \bar{i}}=2 u_{i}^{-3}\left|t_{i}\right|^{2}\left(1+O\left(u_{0}\right)\right)$ and $h_{i \bar{i}}=\frac{1}{2} \frac{u_{i}^{3}}{\left|t_{i}\right|^{2}}\left(1+O\left(u_{0}\right)\right)$ for $1 \leq i \leq m$;

(2) $h^{i \bar{j}}=O\left(\left|t_{i} t_{j}\right|\right)$ and $h_{i \bar{j}}=O\left(\frac{u_{i}^{3} u_{j}^{3}}{\left|t_{i} t_{j}\right|}\right)$, if $1 \leq i, j \leq m$ and $i \neq j$;

(3) $h^{i \bar{j}}=O(1)$ and $h_{i \bar{j}}=O(1)$, if $m+1 \leq i, j \leq n$;

(4) $h^{i \bar{j}}=O\left(\left|t_{i}\right|\right)$ and $h_{i \bar{j}}=O\left(\frac{u_{i}^{3}}{\left|t_{i}\right|}\right)$ if $i \leq m<j$;

(5) $h^{i \bar{j}}=O\left(\left|t_{j}\right|\right)$ and $h_{i \bar{j}}=O\left(\frac{u_{j}^{3}}{\left|t_{j}\right|}\right)$ if $j \leq m<i$

and the Ricci metric has asymptotic

(1) $\tau_{i \bar{i}}=\frac{3}{4 \pi^{2}} \frac{u_{i}^{2}}{\left|t_{i}\right|^{2}}\left(1+O\left(u_{0}\right)\right)$ and $\tau^{i \bar{i}}=\frac{4 \pi^{2}}{3} \frac{\left|t_{i}\right|^{2}}{u_{i}^{2}}\left(1+O\left(u_{0}\right)\right)$, if $i \leq m$;

(2) $\tau_{i \bar{j}}=O\left(\frac{u_{i}^{2} u_{j}^{2}}{\left|t_{i} t_{j}\right|}\left(u_{i}+u_{j}\right)\right)$ and $\tau^{i \bar{j}}=O\left(\left|t_{i} t_{j}\right|\right)$, if $1 \leq i, j \leq m$ and $i \neq j$;

(3) $\tau_{i \bar{j}}=O\left(\frac{u_{i}^{2}}{\left|t_{i}\right|}\right)$ and $h^{i \bar{j}}=O\left(\left|t_{i}\right|\right)$, if $i \leq m<j$;

(4) $\tau_{i \bar{j}}=O(1)$ and $\tau^{i \bar{j}}=O(1)$, if $m+1 \leq i, j \leq n$.

We will also need the following estimates.

Lemma 3.2. Let $(t, s)$ be the pinching coordinates. We have

(1) $\left\|A_{i}\right\|_{0, \Omega_{c}^{i}}=O\left(\frac{u_{i}}{\left|t_{i}\right|}\right)$ and $\left\|A_{i}\right\|_{0, X \backslash \Omega_{c}^{i}}=O\left(\frac{u_{i}^{3}}{\left|t_{i}\right|}\right)$, if $i \leq m$; 
(2) $\left\|A_{i}\right\|_{0}=O(1)$, if $i \geq m+1$;

(3) $\left\|f_{i \bar{i}}\right\|_{0, \Omega_{c}^{i}}=O\left(\frac{u_{i}^{2}}{\left|t_{i}\right|^{2}}\right)$ and $\left\|f_{\bar{i} \bar{i}}\right\|_{0, X \backslash \Omega_{c}^{i}}=O\left(\frac{u_{i}^{6}}{\left|t_{i}\right|^{2}}\right)$, if $i \leq m$;

(4) $\left\|f_{i \bar{j}}\right\|_{0}=O(1)$, if $i, j \geq m+1$;

(5) $\left\|f_{i \bar{j}}\right\|_{0, \Omega_{c}^{i}}=O\left(\frac{u_{i} u_{j}^{3}}{\left|t_{i} t_{j}\right|}\right)$ and $\left\|f_{i \bar{j}}\right\|_{0, \Omega_{c}^{j}}=O\left(\frac{u_{i}^{3} u_{j}}{\left|t_{i} t_{j}\right|}\right)$ and $\left\|f_{i \bar{j}}\right\|_{0, X \backslash\left(\Omega_{c}^{i} \cup \Omega_{c}^{j}\right)}=$ $O\left(\frac{u_{i}^{3} u_{j}^{3}}{\left|t_{i} t_{j}\right|}\right)$ if $i, j \leq m$ and $i \neq j$

(6) $\left\|f_{i \bar{j}}\right\|_{0, \Omega_{c}^{i}}=O\left(\frac{u_{i}}{\left|t_{i}\right|}\right)$ and $\left\|f_{i \bar{j}}\right\|_{0, X \backslash \Omega_{c}^{i}}=O\left(\frac{u_{i}^{3}}{\left|t_{i}\right|}\right)$, if $i \leq m$ and $j \geq m+1$;

(7) $\left|f_{i \bar{j}}\right|_{L^{1}}=O(1)$, if $i, j \geq m+1$;

(8) $\left|f_{i \bar{j}}\right|_{L^{1}}=O\left(\frac{u_{i}^{3}}{\left|t_{i}\right|}\right)$, if $i \leq m$ and $j \geq m+1$;

(9) $\left|f_{i \bar{j}}\right|_{L^{1}}=O\left(\frac{u_{i}^{3} u_{j}^{3}}{\left|t_{i} t_{j}\right|}\right)$, if $i, j \leq m$ and $i \neq j$.

Lemma 3.3. Let $j \leq m$. Then $\int_{\Omega_{c_{1}}^{j}} \widetilde{e}_{j \bar{j}} f_{k \bar{k}} d v \geq 0$ and

$$
\int_{\Omega_{c_{1}}^{j}} \widetilde{e}_{j \bar{j}} f_{k \bar{k}} d v= \begin{cases}O\left(\frac{u_{j}^{4}}{\left|t_{j}\right|^{2}}\right) & k>m \\ O\left(\frac{u_{j}^{4}}{\left|t_{j}\right|^{2}} \frac{u_{k}^{6}}{\left|t_{k}\right|^{2}}\right) & k \leq m, k \neq j \\ O\left(\frac{u_{j}^{5}}{\left|t_{j}\right|^{4}}\right) & k=j\end{cases}
$$

where $\left\{\widetilde{e}_{i \bar{j}}\right\}$ are the functions constructed in [4].

Furthermore, we have

Lemma 3.4. Let $\widetilde{e_{i j}}$ be the functions constructed above. Then

(1) $e_{i \bar{i}}=\widetilde{e_{i \bar{i}}}+O\left(\frac{u_{i}^{4}}{\left|t_{i}\right|^{2}}\right)$, if $i \leq m$;

(2) $e_{i \bar{j}}=\widetilde{e_{i \bar{j}}}+O\left(\frac{u_{i}^{3} u_{j}^{3}}{\left|t_{i} t_{j}\right|}\right)$, if $i, j \leq m$ and $i \neq j$;

(3) $e_{i \bar{j}}=\widetilde{e_{i \bar{j}}}+O\left(\frac{u_{i}^{3}}{\left|t_{i}\right|}\right)$, if $i \leq m$ and $j \geq m+1$;

(4) $\left\|e_{i \bar{j}}\right\|_{0}=O(1)$, if $i, j \geq m+1$.

In $[6]$ we showed

Theorem 3.5. The metric $\widetilde{h}$ on $E$ induced by the WP metric $h$ is good in the sense of Mumford.

Now we are ready to prove the main theorem 3.1. 
Proof. Let $\widetilde{\tau}$ and $\widetilde{\tau}_{C}$ be the metrics on $E$ induced by the Ricci and perturbed Ricci metrics. Since the proof of goodness of these two metrics are similar we will only show that $\widetilde{\tau}$ is good.

We note that, the zero order estimate, namely the first condition in Proposition 2.2 follows from Theorem 3.4 directly. We let $\Gamma_{i p}^{k}$ and $\widetilde{\Gamma}_{i p}^{k}$ be the Christoffell symbols of the WP and Ricci metrics respectively. By Theorem 3.5, since the metric $\widetilde{h}$ on $E$ induced by the WP metric is good, we know that $\left\{\Gamma_{i p}^{k}\right\}$ satisfy the second and third conditions in Proposition 2.2.

To verify the first order estimates for $\widetilde{\tau}$, we only need to show that

$$
\left|\frac{\rho_{i}}{\rho_{k}}\left(\widetilde{\Gamma}_{i p}^{k}-\Gamma_{i p}^{k}\right)\right|=O\left(\Lambda_{p}\right) .
$$

By Theorem 2.3 the above estimate is equivalent to

$$
\left|\frac{\rho_{i}}{\rho_{k}} \tau^{k \bar{j}} h^{\alpha \bar{\beta}}\left\{\sigma_{1} \int_{X_{s}}\left(\xi_{p}\left(e_{i \bar{j}}\right) e_{\alpha \bar{\beta}}\right) d v\right\}\right|=O\left(\Lambda_{p}\right)
$$

for all $1 \leq i, k, p \leq n$.

By using Theorem 3.4, a direct computation shows that, in the sum of the left side of the above formula, if $k \neq j$ or $\alpha \neq \beta$, then the norm of that term is of order $O\left(\Lambda_{p}\right)$. The conclusion follows from the estimate

$$
\int_{X_{s}} \xi_{i}\left(e_{i \bar{i}}\right) e_{i \bar{i}} d v=-\frac{u_{i}^{6}}{32 \pi^{3}\left|t_{i}\right|^{4} t_{i}}\left(1+O\left(u_{0}\right)\right)
$$

which was established in the proof of Theorem 4.4 of [4].

Finally we look at the second order estimate. Let $\widetilde{R}_{i \bar{j} k \bar{l}}$ be the curvature of the Ricci metric. In [4] and [5] we showed that

$$
\left|\widetilde{R}_{i \bar{j} \bar{k} l}\right|=O\left(\Lambda_{i} \Lambda_{j} \Lambda_{k} \Lambda_{l}\right) .
$$

By using this estimate and Theorem 3.4 we know that the last estimate of equations (2.5) hold for $k>m$ or $i=k \leq m$. The remain case follows from the estimate

$$
\left|\widetilde{R}_{i \bar{j} k \bar{l}}\right|=O\left(\Lambda_{i} \Lambda_{j} \Lambda_{k} \Lambda_{l} u_{j}\right)
$$

when $j \leq m$ and $i \neq j$. Again the above refined estimate follows from the proof of Theorem 4.4 and Lemma 4.10 of [4]. 


\section{Naturality of the Ricci Metric}

In this section we prove the restriction property of the Ricci metric. We let $\mathcal{M}_{g}$ be the moduli space of genus $g$ curves where $g \geq 2$ and let $\overline{\mathcal{M}}_{g}$ be its DeligneMumford compactification. We fix a point $p \in \overline{\mathcal{M}}_{g} \backslash \mathcal{M}_{g}$ and let $X=X_{p}$ be the corresponding stable nodal curve. The moduli space $\mathcal{M}(X)$ of the nodal surface $X$ is embedded into $\overline{\mathcal{M}}_{g}$ holomorphically. Furthermore, since each element $Y$ in $\mathcal{M}(X)$ corresponds to a hyperbolic Riemann surface, there is a unique complete Kähler-Einstein metric on $Y$ whose Ricci curvature is -1 .

By the discussion in Section 2 there is a natural WP metric $\hat{h}$ on $\mathcal{M}(X)$. The curvature formula (2.7) is still valid for this WP metric and it is easy to see that the Ricci curvature of the WP metric $\hat{h}$ is negative. We can take $-\operatorname{Ric}\left(\omega_{\hat{h}}\right)$ to be the Kähler form of a Kähler metric on $\mathcal{M}(X)$. This the the Ricci metric $\hat{\tau}$ on $\mathcal{M}(X)$.

In [7] Masur showed that the WP metric $h$ on $\mathcal{M}_{g}$ extends to $\overline{\mathcal{M}}_{g}$ and its restriction to $\mathcal{M}(X)$ via the natural embedding $\mathcal{M}(X) \hookrightarrow \overline{\mathcal{M}}_{g}$ coincides with the WP metric $\hat{h}$ on $\mathcal{M}(X)$. We now describe this phenomenon in local coordinates.

Let $p \in \overline{\mathcal{M}}_{g} \backslash \mathcal{M}_{g}$ be a boundary point of codimension $m$ and let $X=X_{p}$. Let $(t, s)=\left(t_{1}, \cdots, t_{m}, s_{m+1}, \cdots, s_{n}\right)$ be the local pinching coordinates around $p$ where $n=3 g-3$ and $t_{1}, \cdots, t_{m}$ represent the degeneration directions. By the construction in Section 2 we know that $s=\left(s_{m+1}, \cdots, s_{n}\right)$ are local holomorphic coordinates on $\mathcal{M}(X)$ around $p$. The naturality of the WP metric is equivalent to

$$
\lim _{t \rightarrow 0} h_{i \bar{j}}(t, s)=h_{i \bar{j}}(0, s)=\hat{h}_{i \bar{j}}(s)
$$

for any $i, j>m$.

The main result of this section is the naturality of the Ricci metric.

Theorem 4.1. Let $\tau$ be the Ricci metric on the moduli space of genus $g$ curves where $g \geq 2$. Then $\tau$ extends to $\overline{\mathcal{M}}_{g}$. Furthermore, let $p \in \overline{\mathcal{M}}_{g} \backslash \mathcal{M}_{g}$ be a boundary point and let $X=X_{p}$ be the corresponding stable nodal curve. Let $\hat{\tau}$ be the Ricci metric on $\mathcal{M}(X)$. Then the restriction of $\tau$ on $\mathcal{M}(X)$ via the natural embedding $\mathcal{M}(X) \hookrightarrow \overline{\mathcal{M}}_{g}$ coincides with $\hat{\tau}$.

We will need some technical results in [6]. 
Proposition 4.1. Let $(t, s)$ be local pinching coordinates. We have

$$
\left|R_{k \bar{j} p \bar{q}}\right|= \begin{cases}O\left(\Lambda_{p} \Lambda_{q} \frac{u_{j}^{3}}{\left|t_{j}\right|}\right) & j \leq m, k>m \\ O\left(\Lambda_{p} \Lambda_{q}\right) & j>m, k>m \\ O\left(\Lambda_{p} \Lambda_{q} \frac{u_{j}^{3}}{\left|t_{j} t_{k}\right|}\right) & j \leq m, k \leq m \\ O\left(\Lambda_{p} \Lambda_{q} \frac{u_{k}^{3}}{\left|t_{k}\right|}\right) & j>m, k \leq m .\end{cases}
$$

Consequently, we have

$$
\left|\frac{\rho_{i}}{\rho_{k}} R_{i p \bar{q}}^{k}\right|=O\left(\Lambda_{p} \Lambda_{q}\right)
$$

Proof. We fix a local pinching coordinate chart

$$
(U, t, s)=\left(U, t_{1}, \cdots, t_{m}, s_{m+1}, \cdots, s_{n}\right)
$$

around $p$ as in the above discussion and use $s$ as coordinates on $\mathcal{M}(X)$ around $p$. Let $R$ and $\hat{R}$ be the curvature of the WP metrics $h$ and $\hat{h}$ on $\mathcal{M}_{g}$ and $\mathcal{M}(X)$ respectively. We have

$$
\tau_{i \bar{j}}=-h^{k \bar{l}} R_{i \bar{j} k \bar{l}}=-\sum_{k, l>m} h^{k \bar{l}} R_{i \bar{j} k \bar{l}}-\sum_{k \leq m<l} h^{k \bar{l}} R_{i \bar{j} k \bar{l}}-\sum_{l \leq m<k} h^{k \bar{l}} R_{i \bar{j} k \bar{l}}-\sum_{k, l \leq m} h^{k \bar{l}} R_{i \bar{j} k \bar{l}} .
$$

In the rest part of this proof we assume $i, j>m$. Firstly by using Corollary 3.4 and Proposition 4.1 we have

$$
\left|\sum_{k \leq m<l} h^{k \bar{l}} R_{i \bar{j} k \bar{l}}\right|=O\left(u_{k}^{3}\right) \quad \text { and } \quad\left|\sum_{l \leq m<k} h^{k \bar{l}} R_{i \bar{j} k \bar{l}}\right|=O\left(u_{l}^{3}\right) .
$$

Similarly we also have

$$
\left|\sum_{k \neq l \leq m} h^{k \bar{l}} R_{i \bar{j} k \bar{l}}\right|=O\left(u_{l}^{3}\right)
$$

Now we estimate $\left|R_{i \bar{j} k \bar{k}}\right|$ when $k \leq m$. Let $X=X_{t, s}$. By the curvature formula (2.7) we have

$$
\text { (4.5) }\left|R_{i \bar{j} k \bar{k}}\right|=\left|\int_{X}\left(e_{i \bar{k}} f_{k \bar{j}}+e_{k \bar{k}} f_{i \bar{j}}\right) d v\right| \leq\left|\int_{X} e_{i \bar{k}} f_{k \bar{j}} d v\right|+\left|\int_{X} e_{k \bar{k}} f_{i \bar{j}} d v\right| .
$$

To estimate the first term on the right side of the above formula, we first note that if $e \in C^{\infty}(X, \mathbb{C})$ is a complex valued smooth function and $(\square+1) e=f$, 
then $\|e\|_{0} \leq 2\|f\|_{0}$. This follows from applying the maximum principle to the real and imaginary parts of $e$ directly. By using Lemma 3.2 we have

$$
\begin{aligned}
\left|\int_{X} e_{i \bar{k}} f_{k \bar{j}} d v\right| & \leq\left\|e_{i \bar{k}}\right\|_{0}\left|f_{k \bar{j}}\right|_{L^{1}} \leq 2\left\|f_{i \bar{k}}\right\|_{0}\left|f_{k \bar{j}}\right|_{L^{1}} \\
& =O\left(\frac{u_{k}}{\left|t_{k}\right|}\right) O\left(\frac{u_{k}^{3}}{\left|t_{k}\right|}\right)=O\left(\frac{u_{k}^{4}}{\left|t_{k}\right|^{2}}\right) .
\end{aligned}
$$

To estimate the second term on the right side of formula (4.5) we have

$$
\left|\int_{X} e_{k \bar{k}} f_{i \bar{j}} d v\right| \leq\left|\int_{X} \widetilde{e}_{k \bar{k}} f_{i \bar{j}} d v\right|+\left|\int_{X}\left(\widetilde{e}_{k \bar{k}}-e_{k \bar{k}}\right) f_{i \bar{j}} d v\right| .
$$

By Lemma 3.3 and the fact that the support of the function $\widetilde{e}_{k \bar{k}}$ is in $\Omega_{c}^{k}$, we have

$$
\begin{aligned}
\left|\int_{X} \widetilde{e}_{k \bar{k}} f_{i \bar{j}} d v\right| & \leq\left|\int_{\Omega_{c_{1}}^{k}} \widetilde{e}_{k \bar{k}} f_{i \bar{j}} d v\right|+\left|\int_{\Omega_{c}^{k} \backslash \Omega_{c_{1}}^{k}} \widetilde{e}_{k \bar{k}} f_{i \bar{j}} d v\right| \\
& =O\left(\frac{u_{k}^{4}}{\left|t_{k}\right|^{2}}\right)+\left|\int_{\Omega_{c}^{k} \backslash \Omega_{c_{1}}^{k}} \widetilde{e}_{k \bar{k}} f_{i \bar{j}} d v\right| .
\end{aligned}
$$

Since the function $\widetilde{e}_{k \bar{k}}$ is real and nonnegative, by direct computations we have

$$
\left|\int_{\Omega_{c}^{k} \backslash \Omega_{c_{1}}^{k}} \widetilde{e}_{k \bar{k}} f_{i \bar{j}} d v\right| \leq\left\|f_{\overline{i j}}\right\|_{0, \Omega_{c}^{k} \backslash \Omega_{c_{1}}^{k}} \int_{\Omega_{c}^{k} \backslash \Omega_{c_{1}}^{k}} \widetilde{e}_{k \bar{k}} d v=O\left(\frac{u_{k}^{4}}{\left|t_{k}\right|^{2}}\right) .
$$

By combining the above two formulas we get

$$
\left|\int_{X} \widetilde{e}_{k \bar{k}} f_{i \bar{j}} d v\right|=O\left(\frac{u_{k}^{4}}{\left|t_{k}\right|^{2}}\right) .
$$

By Lemma 3.4 we have

$$
\left|\int_{X}\left(\widetilde{e}_{k \bar{k}}-e_{k \bar{k}}\right) f_{i \bar{j}} d v\right| \leq\left\|\widetilde{e}_{k \bar{k}}-e_{k \bar{k}}\right\|_{0}\left|f_{i \bar{j}}\right|_{L^{1}}=O\left(\frac{u_{k}^{4}}{\left|t_{k}\right|^{2}}\right) .
$$

By combining formulas (4.5), (4.6), (4.7), (4.8) and (4.9) we know that

$$
\left|R_{i \bar{j} k \bar{k}}\right|=O\left(\frac{u_{k}^{4}}{\left|t_{k}\right|^{2}}\right)
$$

when $k \leq m$. Thus by Corollary (3.4) we have

$$
\left|\sum_{k \leq m} h^{k \bar{k}} R_{i \bar{j} k \bar{k}}\right|=O\left(u_{k}\right) .
$$


Now we combine estimates (4.3), (4.4) and (4.10) we conclude that

$$
\lim _{t \rightarrow 0} \tau_{i \bar{j}}(t, s)=\lim _{t \rightarrow 0}\left(-\sum_{k, l>m} h^{k \bar{l}} R_{i \bar{j} k \bar{l}}\right)(t, s) .
$$

By definition of the Ricci metric $\hat{\tau}$ on $\mathcal{M}(X)$ we know that

$$
\hat{\tau}_{i \bar{j}}(s)=-\sum_{k, l>m} \hat{h}^{k \bar{l}} \hat{R}_{i \bar{j} k \bar{l}}(s) .
$$

By the work of Masur we know that $\lim _{t \rightarrow 0} h^{k \bar{l}}(t, s)=\hat{h}^{k \bar{l}}(s)$. Thus by combining formulas (4.11) and (4.12), to prove the naturality of the Ricci metric we only need to show that

$$
\lim _{t \rightarrow 0} R_{i \bar{j} k \bar{l}}(t, s)=\hat{R}_{i \bar{j} k \bar{l}}(s)
$$

for all $i, j, k, l>m$. By using the curvature formula (2.7) we only need to show that

$$
\lim _{t \rightarrow 0}\left(\int_{X_{t, s}}\left(e_{i \bar{j}} f_{k \bar{l}}+e_{i \bar{l}} f_{k \bar{j}}\right) d v\right)(t, s)=\left(\int_{X_{0, s}}\left(e_{i \bar{j}} f_{k \bar{l}}+e_{i \bar{l}} f_{k \bar{j}}\right) d v\right)(s) .
$$

However, the above formula follows from estimates (3.2) and the fact that $\left|B_{k}(z)\right| \rightarrow$ 0 as $z$ approach the cusp on a nodal surface.

\section{REFERENCES}

[1] L. Ahlfors and L. Bers. Riemann's mapping theorem for variable metrics. Ann. of Math. (2), 72:385-404, 1960.

[2] P. Deligne and D. Mumford. The irreducibility of the space of curves of given genus. Inst. Hautes Études Sci. Publ. Math., (36):75-109, 1969.

[3] L. Keen. Collars on Riemann surfaces. In Discontinuous groups and Riemann surfaces (Proc. Conf., Univ. Maryland, College Park, Md., 1973), pages 263-268. Ann. of Math. Studies, No. 79. Princeton Univ. Press, Princeton, N.J., 1974.

[4] K. Liu, X. Sun, and S.-T. Yau. Canonical metrics on the moduli space of Riemann surfaces. I. J. Differential Geom., 68(3):571-637, 2004.

[5] K. Liu, X. Sun, and S.-T. Yau. Canonical metrics on the moduli space of Riemann surfaces. II. J. Differential Geom., 69(1):163-216, 2005.

[6] K. Liu, X. Sun, and S.-T. Yau. Good geometry on the curve moduli. Publ. Res. Inst. Math. Sci., 44(2):699-724, 2008.

[7] H. Masur. Extension of the Weil-Petersson metric to the boundary of Teichmuller space. Duke Math. J., 43(3):623-635, 1976.

[8] D. Mumford. Hirzebruch's proportionality theorem in the noncompact case. Invent. Math., 42:239-272, 1977. 
[9] S. Trapani. On the determinant of the bundle of meromorphic quadratic differentials on the Deligne-Mumford compactification of the moduli space of Riemann surfaces. Math. Ann., 293(4):681-705, 1992.

[10] S. A. Wolpert. Chern forms and the Riemann tensor for the moduli space of curves. Invent. Math., 85(1):119-145, 1986.

[11] S. A. Wolpert. Asymptotics of the spectrum and the Selberg zeta function on the space of Riemann surfaces. Comm. Math. Phys., 112(2):283-315, 1987.

[12] S. A. Wolpert. The hyperbolic metric and the geometry of the universal curve. J. Differential Geom., 31(2):417-472, 1990.

Kefeng Liu

Center of Mathematical Sciences, Zhejiang University, Zhejiang 310027, China.

Department of Mathematics, University of California, Los Angeles, USA.

E-mail: liu@math.ucla.edu

Xiaofeng Sun

Department of Mathematics, Lehigh University, Bethlehem, PA 18015, USA.

E-mail: xis205@lehigh.edu

Shing-Tung Yau

Department of Mathematics, Harvard University, One Oxford Street, Cambridge, MA 02138, USA.

E-mail: yau@math.harvard.edu 